\title{
Dynamically evolving Mg II broad absorption line flow in SDSS J133356.02+001229.1
}

\author{
M. Vivek, ${ }^{1 \star}$ R. Srianand,${ }^{2}$ A. Mahabal ${ }^{3}$ and V. C. Kuriakose ${ }^{1}$ \\ ${ }^{1}$ Department of Physics, Cochin University of Science and Technology, Kochi 682022, India \\ ${ }^{2}$ Inter-University Centre for Astronomy and Astrophysics, Pune 410007, India \\ ${ }^{3}$ Caltech, MC 249-17, Pasadena, CA 91125, USA
}

Accepted 2011 December 30. Received 2011 November 26; in original form 2011 October 30

\begin{abstract}
We report a dynamically evolving low-ionization broad absorption line (BAL) flow in the QSO SDSS J133356.02+001229.1 (at $z_{\mathrm{em}} \sim 0.9197$ ). These observations are part of our ongoing monitoring of low-ionization BAL QSOs with the 2-m telescope at IUCAA Girawali Observatory (IGO). The broad Mg II absorption with an ejection velocity of $1.7 \times 10^{4} \mathrm{~km} \mathrm{~s}^{-1}$, found in the Sloan Digital Sky Survey (SDSS) spectra, has disappeared completely in our IGO spectra. We found an emerging new component at an ejection velocity of $2.8 \times 10^{4} \mathrm{~km} \mathrm{~s}^{-1}$. During our monitoring period this component has shown strong evolution both in its velocity width and optical depth and nearly disappeared in our latest observations. Acceleration of a low-velocity component seen in SDSS spectrum to a higher velocity is unlikely as the $\mathrm{Mg}$ II column densities are always observed to be higher for the new component. We argue that the observed variations may not be related to ionization changes and are consistent with absorption produced by multistreaming flow transiting across our line of sight. We find a possible connection between flux variation of the QSO and $N\left(\mathrm{Mg}_{\text {II }}\right)$ of the newly emerged component. This could mean the ejection being triggered by changes in the accretion disc or dust reddening due to the outflowing gas.
\end{abstract}

Key words: galaxies: active - quasars: absorption lines - quasars: general - quasars: individual: J133356.02+001229.1.

\section{INTRODUCTION}

It is widely believed that the kinetic energy output from quasars through the outflows are as important as their radiative output. They are believed to play an important role in regulating the growth of supermassive black holes (Silk \& Rees 1998; King 2003) and star formation in the host galaxies (e.g. Bower et al. 2006). In addition, outflows influence the enrichment of the surrounding intergalactic medium. Therefore, it is important to understand the origin and evolution of QSO outflows. Large-scale outflows manifest itself in the form of broad absorption lines (BALs) in QSO spectra. BAL QSOs comprise of up to 40 per cent of the total QSO population (Dai, Shankar \& Sivakoff 2008, and references therein). The correct explanation for the observed incidence of BAL QSOs is still a subject of debate between the orientational (see e.g. Goodrich \& Miller 1995; Hines \& Wills 1995; Murray et al. 1995; Elvis 2000) and evolutionary models (see e.g. Hazard et al. 1984; Becker et al. 2000).

Line variability studies of BAL QSOs are useful for understanding the physical conditions and dynamics of the gas close

${ }^{\star}$ E-mail: vivekm@iucaa.ernet.in to the central engine. The time variability of $\mathrm{C}$ IV and Si IV absorption is reported in several cases (e.g. Srianand \& Petitjean 2001; Lundgren et al. 2007; Gibson et al. 2008). Such a variability could be related either to variations in the ionization state or to the covering factor of the absorbing gas. However, the most interesting cases are the ones where the flow emerges afresh or shows strong dynamical evolution (i.e. variation in the absorption profile and signatures of acceleration). There are five previous reports of emerging $\mathrm{C}$ IV BAL discovery in the literature [TEX 1726+344 (Ma 2002), SDSS J105400.40+034801.2 (Hamann et al. 2008), WPVS 007 (Leighly et al. 2009), Ton 34 (Krongold, Binette \& Hernández-Ibarra 2010) and PG0935+417 (Rodríguez Hidalgo, Hamann \& Hall 2011)]. These studies attribute the observed dynamical evolution to multiple streaming wind moving across the line of sight. Detecting an emerging $\mathrm{Mg}$ II flow will be very interesting as $\mathrm{Mg}$ II BALs are considered to be a possible manifestation of a QSO's efforts to expel a thick shroud of gas and dust (Voit, Weymann \& Korista 1993).

Here, we report the first discovery of an emerging $\mathrm{Mg}$ II outflow in a QSO SDSS J133356.02+001229.1 (hereafter J1333+0012) at a redshift $z_{\mathrm{em}} \sim 0.9197$ (Hewett, Foltz \& Chaffee 2001; Trump et al. 2006). This source is part of our sample of low-ionization BAL QSOs that are being spectroscopically monitored at IUCAA Girawali Observatory (IGO). Our spectra revealed the emergence of 
Table 1. Log of observations.

\begin{tabular}{cccccccc}
\hline Source & Instrument & Date & MJD & $\begin{array}{c}\text { Exposure time } \\
(\mathrm{min})\end{array}$ & $\begin{array}{c}\lambda \text { coverage } \\
(\AA)\end{array}$ & $\begin{array}{c}\text { Resolution } \\
\left(\mathrm{km} \mathrm{s}^{-1}\right)\end{array}$ & ${\mathrm{S} / \mathrm{N}^{a}}^{a}$ \\
\hline \multirow{5}{*}{ J1333+0012 } & SDSS & 2000 April 28 & 51662 & $45 \times 1$ & $3800-9200$ & 150 & 27 \\
& SDSS & 2001 February 15 & 51955 & $105 \times 1$ & $3800-9200$ & 150 & 40 \\
& IGO/IFOSC 7 & 2008 April 3 & 54559 & $40 \times 2$ & $3800-6840$ & 300 & 11 \\
& IGO/IFORS 1 & 2009 February 26 & 54888 & $45 \times 3$ & $3270-6160$ & 360 & 21 \\
& IGO/IFOSC 7 & 2009 March 26 & 54916 & $45 \times 3$ & $3800-6840$ & 300 & 12 \\
& IGO/IFOSC 7 & 2009 April 19 & 54940 & $45 \times 2$ & $3800-6840$ & 300 & 5 \\
& IGO/IFORS 1 & 2010 January 22 & 55218 & $45 \times 1$ & $3270-6160$ & 360 & 19 \\
& IGO/IFORS 1 & 2010 January 25 & 55221 & $45 \times 1$ & $3270-6160$ & 360 & 16 \\
& IGO/IFORS 1 & 2010 March 14 & 55269 & $45 \times 3$ & $3270-6160$ & 360 & 16 \\
& IGO/IFOSC 7 & 2011 April 6 & 55657 & $45 \times 3$ & $3800-6840$ & 300 & 19 \\
\hline
\end{tabular}

${ }^{a}$ Calculated over the wavelength range 5800-6200 A.

a new $\mathrm{Mg}$ II broad absorption component that was not present in the Sloan Digital Sky Survey (SDSS) spectra obtained 7 years earlier. We have been monitoring this new $\mathrm{Mg}$ II absorption component for the past 4 years. In Section 2, we provide details of the observations and data reduction. Results and discussions are presented in Sections 3 and 4, respectively.

\section{OBSERVATION AND DATA REDUCTION}

Our spectroscopic observations were carried out with IUCAA Faint Object Spectrograph (IFOSC) mounted on a 2-m telescope at IGO. The details of these observations together with that of the archival SDSS spectra are given in Table 1. We have used Grism \#1 and Grism \#7 of IFOSC in combination with a long slit having a width of $1.5 \mathrm{arcsec}$. This combinations have the wavelength coverage of 3270-6160 and 3800-6840 A, respectively. Typically the observations were split into exposures of $45 \mathrm{~min}$. Spectra obtained on closely separated dates were combined for better signal-to-noise ratio. Cleaning of the raw frames and 1D spectral extraction was carried out using the standards procedures in IRAF. ${ }^{1}$ We opted for the variance-weighted extraction with 'doslit' procedure. Wavelength calibrations were done using standard helium-neon lamp spectra, and flux calibrations were done using a standard star spectra observed on the same night. Air-to-vacuum conversion was applied before adding the spectra. Individual spectra were combined using $1 / \sigma^{2}$ weighting in each pixel after scaling the overall individual spectra within a sliding window. The error spectrum was computed taking into account proper error propagation during the combining process.

Fig. 1 shows the SDSS spectrum taken on 2001 February 15. The expected positions of broad emission lines (at $z_{\mathrm{em}}=0.9197$ ) are marked with blue dotted vertical lines. Narrow $\mathrm{Mg}_{\text {II }}, \mathrm{Mg}_{\text {I }}$ and $\mathrm{Fe}_{\text {II }}$ absorption lines associated with the intervening absorbers at $z_{\text {abs }} \sim 0.898$ and 0.836 are distinctly detected. In addition, two broad Mg II absorption components (marked in Fig. 1 as ' $\mathrm{B}$ ' and ' $R$ ' to denote the blue and red components, respectively) are clearly detected. We do not detect any associated Fe II lines, and the $\mathrm{Al}$ III lines are redshifted out of the coverage. A visual comparison of this spectrum with the Fe template of Vestergaard \& Wilkes (2001) suggests that the continuum of $\mathrm{J} 1333+0012$ may have considerable contribution from broad $\mathrm{Fe}$ emission lines.

\footnotetext{
${ }^{1}$ IRAF is distributed by the National Optical Astronomy Observatories, which are operated by the Association of Universities for Research in Astronomy, Inc., under cooperative agreement with the National Science Foundation.
}

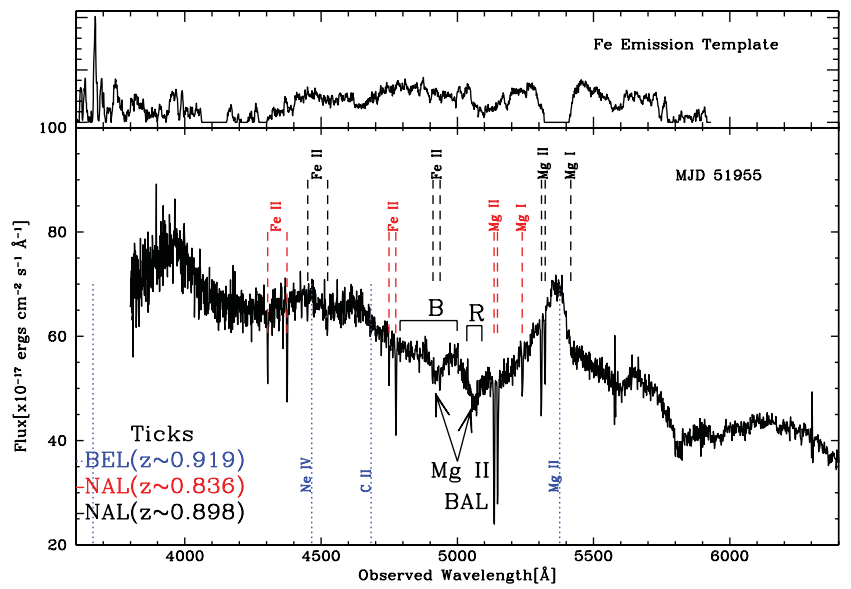

Figure 1. The SDSS spectrum of J1333+0012. The expected positions of broad emission lines are marked with blue dotted lines. The narrow absorption lines at $z_{\text {abs }} \sim 0.898$ and 0.836 are shown by black and red dashed lines. Arrows show the red (marked as R) and blue (marked as B) components of $\mathrm{Mg}$ II BAL discussed in this Letter. Fe emission template from Vestergaard \& Wilkes (2001) is shown in the upper panel for comparison.

\section{RESULTS}

Trump et al. (2006) have fitted the spectrum of J1333+0012, observed on 2001 February 15, using a template spectrum. Their bestfitting template resulted in a spectral index $\left(f_{v} \propto v^{\alpha_{v}}\right)$ of $\alpha_{v}=1.64$ and $E(B-V)=0.295$ for a SMC-type dust. They have identified only one distinct BAL component at $z_{\text {abs }}=0.811$ (corresponding to $\mathrm{R}$ component defined above). They assigned a balnicity index and absorption index of $36 \pm 0.42$ and $459 \pm 0.75$, respectively, to this source. Apart from this $\mathrm{R}$ component [that has consistent profile between two SDSS spectra and the Keck spectra of Hewett et al. (2001) observed on 2000 April 30], we find a new component at $z_{\text {abs }}=0.764$ (defined above as B) in the 2001 SDSS data (not clearly detected in the previous epoch SDSS spectrum) having a velocity width of $\sim 6000 \mathrm{~km} \mathrm{~s}^{-1}$.

In Fig. 2, the IGO spectra obtained at different epochs are compared with a reference SDSS spectrum obtained in the year 2000 (i.e. MJD 51662). For displaying purpose, we have scaled the fluxes to match the flux of the reference spectrum. In the bottom half of each panel, we show the ratio of spectrum obtained at a given epoch to that of the reference spectrum. This curve is smooth apart from the wavelength range of $\mathrm{R}$ and $\mathrm{B}$ components. We normalize this curve with a smooth lower order polynomial (shown as red dotted 

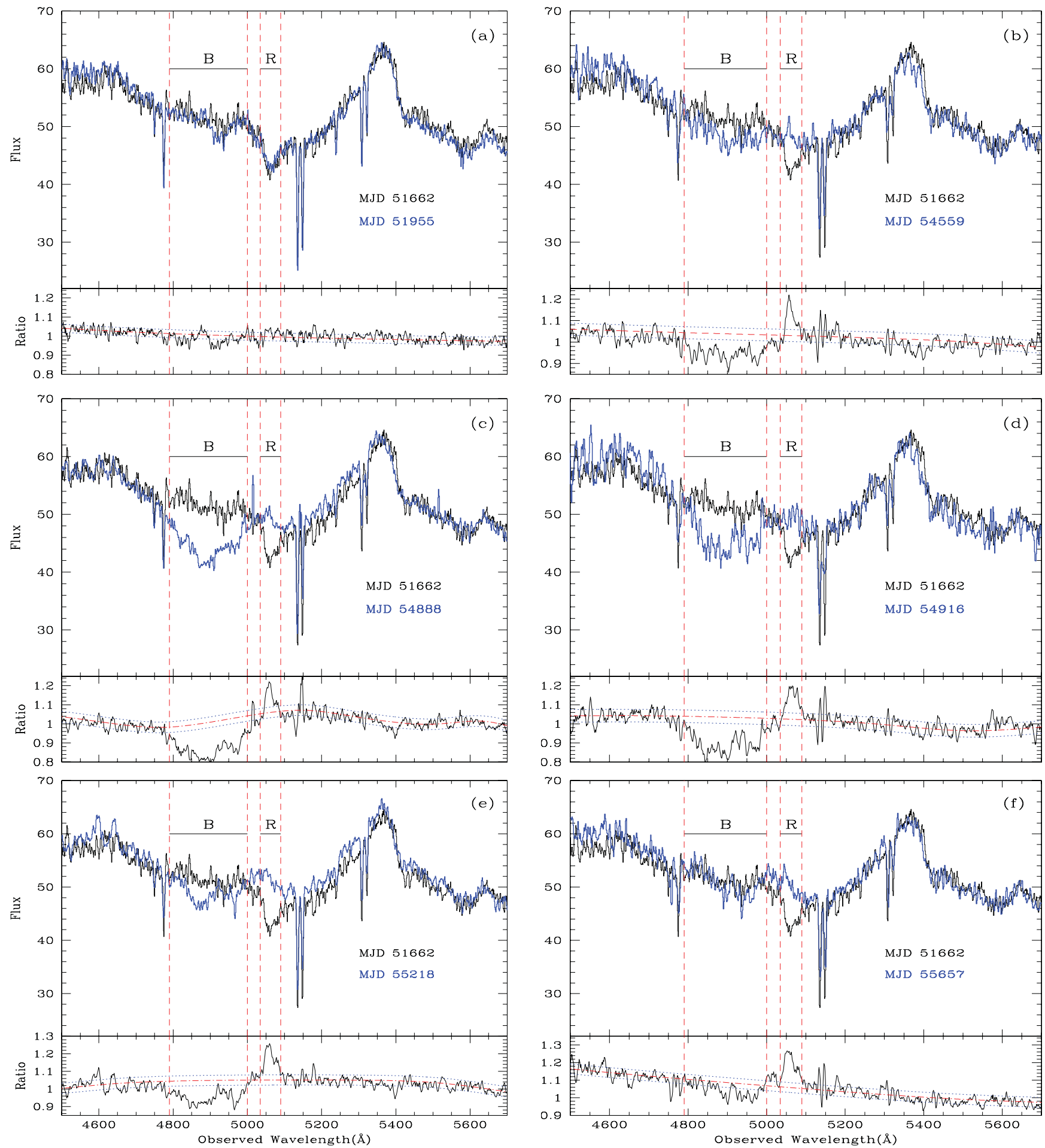

Figure 2. The spectra of J1333+0012 obtained at different epochs are compared with the initial SDSS spectrum. The observed flux is scaled to match the SDSS flux. The vertical lines show the wavelength range of $\mathrm{R}$ and $\mathrm{B}$ components as in Fig. 1. The ratio between the SDSS and the corresponding IGO spectrum is plotted in the lower panel in each plot. A lower order polynomial fit to this curve and the associated error are also shown.

curve in Fig. 2) to remove any large wavelength scale continuum flux differences between the two spectra.

In our IGO 2008 (MJD 54559) spectrum (see panel b in Fig. 2), the $\mathrm{R}$ component (that was seen with near identical optical depth in two SDSS spectra) has completely disappeared. The R component has never reappeared again in any of our subsequent IGO spectra (see Fig. 2). At the same time, the B component is seen to have varied in depth and widened in velocity in the 2008 (MJD 54559) data. In 2009 (MJD 54888), the B component further deepened in the absorption troughs. However, no changes in the velocity structure are seen (see panels c and d in Fig. 2). It diminished in strength in 2010 and has almost vanished in 2011 (see panels e and $\mathrm{f}$ in Fig. 2). 
Table 2. Column density measurements.

\begin{tabular}{lcccccc}
\hline MJD & \multicolumn{3}{c}{ R component } & \multicolumn{3}{c}{ B component } \\
& $\begin{array}{c}\log N\left(\mathrm{Mg}_{\mathrm{II}}\right)^{1} \\
\left(\mathrm{~cm}^{-2}\right)\end{array}$ & $\begin{array}{c}\Delta v^{a} \\
\left(\mathrm{~km} \mathrm{~s}^{-1}\right)\end{array}$ & $z_{\mathrm{abs}}{ }^{b}$ & $\begin{array}{c}\log N\left(\mathrm{Mg} \mathrm{II}^{1}\right)^{-} \\
\left(\mathrm{cm}^{-2}\right)\end{array}$ & $\begin{array}{c}\Delta v^{a} \\
\left(\mathrm{~km} \mathrm{~s}^{-1}\right)\end{array}$ & $z_{\mathrm{abs}}{ }^{b}$ \\
\hline 51662 & $13.62 \pm 0.20$ & 2829 & 0.811 & $\leq 13.42^{c}$ & 7000 & - \\
51955 & $13.75 \pm 0.13$ & 3934 & 0.812 & $13.36 \pm 0.25$ & 6143 & 0.764 \\
54559 & $\leq 13.57^{c}$ & 3000 & - & $14.26 \pm 0.39$ & 12303 & 0.747 \\
54888 & $\leq 13.20^{c}$ & 3000 & - & $14.50 \pm 0.10$ & 12909 & 0.746 \\
54916 & $\leq 13.42^{c}$ & 3000 & - & $14.46 \pm 0.20$ & 11900 & 0.745 \\
55218 & $\leq 13.09^{c}$ & 3000 & - & $14.28 \pm 0.13$ & 11474 & 0.754 \\
55657 & $\leq 13.35^{c}$ & 3000 & - & $13.76 \pm 0.36$ & 10016 & 0.760 \\
\hline
\end{tabular}

${ }^{1}$ The error in $N(\mathrm{Mg}$ II $)$ does not include the contribution from continuum placement uncertainties; ${ }^{a}$ velocity range that contains 90 per cent of the integrated optical depth (see Ledoux et al. 2006); ${ }^{b}$ optical depth weighted redshift; ${ }^{c} 3 \sigma$ upper limit of $N(\mathrm{Mg}$ II).

We calculated the Mg II column density for both R and B components using apparent optical depth method (Savage \& Sembach 1991) assuming the gas to be optically thin and covering the background source completely. In the case of component $\mathrm{B}$, we have used the normalized ratio spectra discussed in Fig. 2 to estimate $N\left(\mathrm{Mg}_{\mathrm{II}}\right)$. For component $\mathrm{R}$ that is relatively narrow, we use the observed flux in the neighbourhood of the absorption line for continuum normalization. The measured $\mathrm{Mg}$ II column densities (or $3 \sigma$ upper limits), velocity width ( $\Delta v)$ and $z_{\text {abs }}$ for both the components are summarized in Table 2.

In Fig. 3 we plot the photometric light curves of J1333+0012 obtained in the Catalina Real-time Transient Survey (CRTS; Drake et al. 2009). These observations were carried out with an unfiltered set-up and were converted to $V$ magnitude using 10-100 wellselected G-type dwarf calibration stars found in each frame and the method described in Henden (2000). The zero-point uncertainty

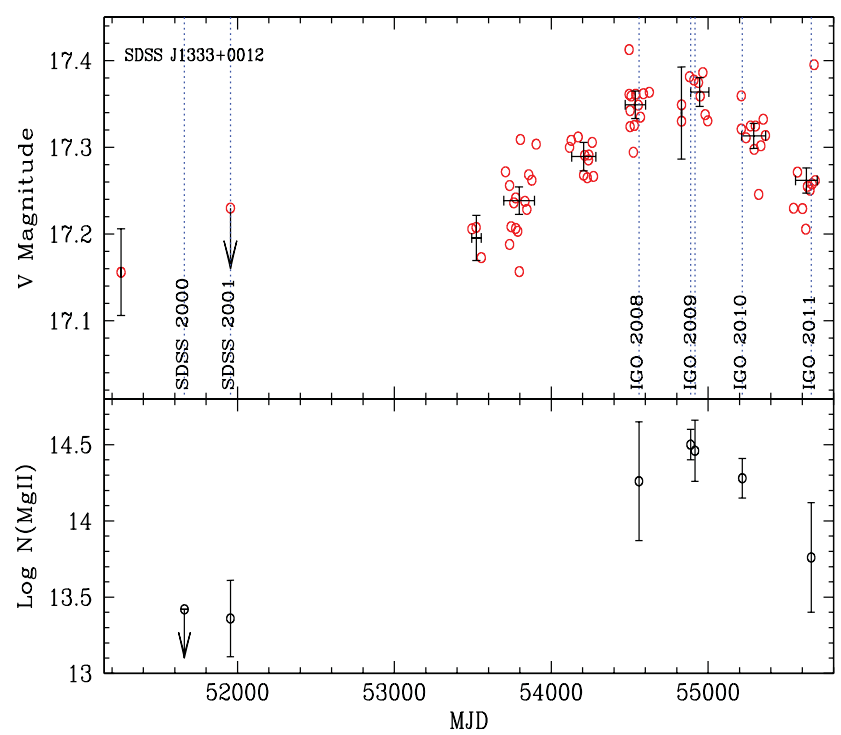

Figure 3. Top panel: light curve (in Johnson's $V$ magnitude) of J1333+0012 from CRTS and SDSS (first two points) measurements. Black points are the average of closely spaced observations, and the associated error is obtained from errors in individual measurements. The error bars along $\mathrm{x}$-axis correspond to the time period over which the individual measurements are averaged. The epochs of spectroscopic observations are marked as blue dotted vertical lines. Bottom panel: the measured $N(\mathrm{Mg}$ II $)$ of the B component versus MJD. is found to be $\leq 0.08$ mag. The two leftmost points in the light curve correspond to the transformed SDSS photometric and fibre magnitudes, respectively. We use the transformation equation given in Jester et al. (2005) for $z \leq 2.1$ QSOs for this. Fibre magnitude should be considered as an upper limit on the actual magnitude. The QSO has dimmed between the early epoch CRTS observations and IGO 2008 observations, reached a minimum in 2009 and then brightened thereafter. The maximum seen amplitude of the variation is $\sim 0.2$ mag. Very interestingly, a similar but opposite variation is seen in $N(\mathrm{Mg}$ II $)$ of the $\mathrm{B}$ component during this period, i.e. when the quasar flux was minimum, the measured $N(\mathrm{Mg}$ II $)$ is maximum.

\section{DISCUSSION}

The main result of our monitoring of $\mathrm{J} 1333+0012$ is that the $\mathrm{R}$ component of $\mathrm{Mg}$ II absorption has disappeared completely after the year 2001 and the B component emerged, became stronger and wider and subsequently disappeared between the years 2001 and 2011. There is also tentative evidence for a possible connection between the QSO flux and $N(\mathrm{Mg}$ II) measured for the B component. Now we discuss various scenarios to explain these observations.

It is very tempting to say that the $\mathrm{R}$ component got accelerated to the B component and later vanished from our line of sight. From Table 2 , it is clear that the values of $N(\mathrm{Mg}$ II $)$ and $\Delta v$ of component $\mathrm{B}$ in later epochs are much higher than that seen in the $\mathrm{R}$ component in earlier epochs. If we use thin shell approximation, then mass conservation suggests that $r^{2} N(\mathrm{H})$ should be conserved, where $r$ and $N(\mathrm{H})$ are the distance of the shell from the QSO and total hydrogen column density, respectively. We expect $r$ to increase with time, so $N(\mathrm{H})$, and hence $N(\mathrm{Mg} I)$, should decrease with time. The observed $N\left(\mathrm{Mg}_{\text {II }}\right)$ values do not support this scenario.

One could argue that there were always two fluid components and what we see is due to the variations in the ionization parameter. Above we have seen that the QSO has varied by $\sim 0.2 \mathrm{mag}$. However, the required change in $N(\mathrm{Mg}$ II $)$ is $0.6-1$ dex. It is clear from fig. 2 of Hamann (1997) that the required change in $N(\mathrm{Mg}$ II cannot be produced by a 0.2 -dex change in ionization parameter. In addition, the change in the $\mathrm{Mg}$ II optical depth of $\mathrm{R}$ and $\mathrm{B}$ components are in the opposite directions. This scenario can be consistent if the variation in UV continuum is much larger than the $0.2 \mathrm{mag}$ seen in the $V$ band and the initial ionization parameters of the two components are very different. We do not favour this scenario based on the lack of large colour variations between our IFOSC spectra and the near consistency of $\mathrm{Mg}$ II emission line.

In all the previous cases of emerging flows (Ma 2002; Hamann et al. 2008; Krongold et al. 2010; Hall et al. 2011; Rodríguez Hidalgo et al. 2011), the most favoured explanation is the transverse motion of the absorbing gas. In particular, the evolution of the B component can be explained as the absorbing gas transiting through our line of sight as the component has emerged, evolved and disappeared. To consider this possibility, we derive some basic parameters for $\mathrm{J} 1333+0012$.

We obtained the $B$-band magnitude of this QSO using the measured $u$ and $g$ magnitudes (see Jester et al. 2005). We use this and the prescription of Marconi et al. (2004) to get the bolometric luminosity, $L_{\mathrm{bol}}=9.39 \times 10^{46} \mathrm{erg} \mathrm{s}^{-1}$. The corresponding mass accretion rate, $\dot{M}_{\text {in }}$, is

$\dot{M}_{\text {in }}=\frac{L_{\text {bol }}}{\epsilon c^{2}}=16.9 \mathrm{M}_{\odot} \mathrm{yr}^{-1}$,

where $\epsilon$, the mass to energy conversion efficiency, is taken as 0.1 . The black hole mass derived from Eddington accretion is $8 \times$ 
$10^{8} \mathrm{M}_{\odot}$. Following Hall et al. (2011), we find the diameter of the disc within which 90 per cent of the 2700 - $\AA$ continuum is emitted to be $D_{2700} \sim 10^{16} \mathrm{~cm}$.

The variability of the $\mathrm{R}$ component has occurred over a period of $3994 \mathrm{~d}$ in the observer's frame or $2081 \mathrm{~d}$ in the rest frame of the QSO. We can use this as an upper limit on the transit time of the absorbing gas across the continuum emitting region. If we assume the projected transverse size of the absorbing gas is much smaller than the emitting region (assumed to be a face-on disc), then we can estimate the transverse velocity $v_{\perp} \geq 550 \mathrm{~km} \mathrm{~s}^{-1}$. We find the radial velocity up to $v_{\mathrm{r}}=2.8 \times 10^{4} \mathrm{~km} \mathrm{~s}^{-1}$ and the radial displacement of the gas during the transit is $\sim 0.1 \mathrm{pc}$. Even if the projected transverse size of the gas is 10 times the continuum emitting region, the expected transverse velocity will be much lower than the measured radial velocity.

The main question is whether the emergence of new component is in any way related to the structural changes in the accretion disc. The possible connection between QSO dimming and $N(\mathrm{Mg}$ II $)$ is interesting. It could either mean the ejection being triggered by some events in the accretion disc that caused reduction in the accretion efficiency or extinction by dust in the transiting gas.

Trump et al. (2006) identified this system as BAL based on the presence of $\mathrm{Mg}$ II absorption in the $\mathrm{R}$ component. The latest spectrum of J1333+0012 taken with IGO is nearly devoid of any broad $\mathrm{Mg}$ II absorption. We do not detect $\mathrm{Mg}_{\text {I }}, \mathrm{Fe}_{\text {II }}$ and $\mathrm{Ca}$ II absorption associated with either of the broad $\mathrm{Mg}$ II components. It will be interesting to study the properties of other strong high-ionization lines using UV spectroscopy. Continuous optical monitoring of this source to see recurrence of new outflow episode will be useful for modelling this interesting source.

\section{CONCLUSIONS}

In this Letter, we report a dynamically evolving $\mathrm{Mg}$ II BAL outflow in SDSS J133356.02+001229.1. The R component detected in the SDSS spectra taken in 2000 and 2001 was never seen in the IGO spectra taken in the latter epochs. The blue component B emerged in 2001. It continued to increase its optical depth till 2009 and started to diminish thereafter. This component has almost vanished in the most recent spectra in 2011.

We provide arguments for not favouring the acceleration of a single cloud to a higher velocity and changes in the ionization conditions causing the observed spectral variability. The observed variability can be best explained by multiple streaming gas moving across our line of sight. An outflowing gas component with transverse velocity one-tenth of the observed radial velocity and flow extent up to 10 times the size of the accretion disc can easily explain these observations.

We find the QSO has dimmed by about $0.2 \mathrm{mag}$ (in $V$ band) during the monitoring period. This may be the manifestation of the emerged outflow being triggered by the structural changes in the accretion disc or due to reddening by dust in the absorbing gas.

\section{ACKNOWLEDGMENTS}

We wish to acknowledge the IUCAA/IGO staff for their support during our observations. In particular, we thank Dr Vijay Mohan for continued support. MV gratefully acknowledges University Grants Commission, India, for support through RFSMS Scheme and IUCAA for hospitality, where most of this work was done. RS gratefully acknowledges the support from the Indo-French Center for the Promotion of Advanced Research (Centre Franco-Indien pour la promition de la recherche avancée) under project N. 4304-2.

\section{REFERENCES}

Becker R. H., White R. L., Gregg M. D., Brotherton M. S., LaurentMuehleisen S. A., Arav N., 2000, ApJ, 538, 72

Bower R. G., Benson A. J., Malbon R., Helly J. C., Frenk C. S., Baugh C. M., Cole S., Lacey C. G., 2006, MNRAS, 370, 645

Dai X., Shankar F., Sivakoff G. R., 2008, ApJ, 672, 108

Drake A. J. et al., 2009, ApJ, 696, 870

Elvis M., 2000, ApJ, 545, 63

Gibson R. R., Brandt W. N., Schneider D. P., Gallagher S. C., 2008, ApJ, 675,985

Goodrich R. W., Miller J. S., 1995, ApJ, 448, L73

Hall P. B., Anosov K., White R. L., Brandt W. N., Gregg M. D., Gibson R. R., Becker R. H., Schneider D. P., 2011, MNRAS, 411, 2653

Hamann F., 1997, ApJS, 109, 279

Hamann F., Kaplan K. F., Rodríguez Hidalgo P., Prochaska J. X., HerbertFort S., 2008, MNRAS, 391, L39

Hazard C., Morton D. C., Terlevich R., McMahon R., 1984, ApJ, 282, 33

Henden A. A., 2000, J. American Association Var. Star Obser., 29, 35

Hewett P. C., Foltz C. B., Chaffee F. H., 2001, AJ, 122, 518

Hines D. C., Wills B. J., 1995, ApJ, 448, L69

Jester S. et al., 2005, AJ, 130, 873

King A., 2003, ApJ, 596, L27

Krongold Y., Binette L., Hernández-Ibarra F., 2010, ApJ, 724, L203

Ledoux C., Petitjean P., Fynbo J. P. U., Møller P., Srianand R., 2006, A\&A, 457, 71

Leighly K. M., Hamann F., Casebeer D. A., Grupe D., 2009, ApJ, 701, 176

Lundgren B. F., Wilhite B. C., Brunner R. J., Hall P. B., Schneider D. P., York D. G., Vanden Berk D. E., Brinkmann J., 2007, ApJ, 656, 73

Ma F., 2002, MNRAS, 335, L99

Marconi A., Risaliti G., Gilli R., Hunt L. K., Maiolino R., Salvati M., 2004, MNRAS, 351, 169

Murray N., Chiang J., Grossman S. A., Voit G. M., 1995, ApJ, 451, 498

Rodríguez Hidalgo P., Hamann F., Hall P., 2011, MNRAS, 411, 247

Savage B. D., Sembach K. R., 1991, ApJ, 379, 245

Silk J., Rees M. J., 1998, A\&A, 331, L1

Srianand R., Petitjean P., 2001, A\&A, 373, 816

Trump J. R. et al., 2006, ApJS, 165, 1

Vestergaard M., Wilkes B. J., 2001, ApJS, 134, 1

Voit G. M., Weymann R. J., Korista K. T., 1993, ApJ, 413, 95

This paper has been typeset from a $\mathrm{T}_{\mathrm{E}} \mathrm{X} / \mathrm{LT} \mathrm{T} \mathrm{X}$ file prepared by the author. 